\title{
Saliency Detection in Images using Graph-based Rarity, Spatial Compactness and Background Prior
}

\author{
Sudeshna Roy and Sukhendu Das \\ Visualization and Perception Lab., Department of Computer Science and Engineering, \\ Indian Institute of Technology, Madras, India \\ sudeshna@cse.iitm.ac.in, sdas@iitm.ac.in
}

\begin{abstract}
Keywords: $\quad$ Saliency, Spectral Clustering, Feature Rarity, Boundary Prior.
Abstract: Bottom-up saliency detection techniques extract salient regions in an image while free-viewing the image. We have approached the problem with three different low-level cues- graph based rarity, spatial compactness and background prior. First, the image is broken into similar colored patches, called superpixels. To measure rarity we represent the image as a graph with superpixels as node and exponential color difference as the edge weights between the nodes. Eigenvectors of the Laplacian of the graph are then used, similar to spectral clustering ( $\mathrm{Ng}$ et al., 2001). Each superpixel is associated with a descriptor formed from these eigenvectors and rarity or uniqueness of the superpixels are found using these descriptors. Spatial compactness is computed by combining disparity in color and spatial distance between superpixels. Concept of background prior is implemented by finding the weighted Mahalanobis distance of the superpixels from the statistically modeled mean background color. These cues in combination gives the proposed saliency map. Experimental results demonstrate that our method outperforms many of the recent state-of-the-art methods both in terms of accuracy and speed.
\end{abstract}

\section{INTRODUCTION}

Visual Saliency have now a days become very popular and relevant way to deal with a lot of computer vision tasks like object detection, object recognition, content based image retrieval, scene understating etc. It reduces the search space of the problem, as well as helps in extracting correct features for these tasks. It is a perceptual quality of the human visual system, by which humans attend to a subset of the pool of available visual information. Saliency of an image is given by a saliency map where we assign a normalized value to an image component or superpixel denoting its probability of being salient. A salient region in an image is sufficiently distinct from its neighborhood in terms of visual attributes or features, and grabs attention. In this paper, we concentrate on unsupervised bottom-up saliency detection technique when free-viewing a scene. Bottom-up saliency can be thought as a filter which extracts selected spatial locations of interest which generally stands out from other locations. Most work in the past have defined saliency by, either using spatial features like color, orientation, spatial distances between image patches (Itti et al., 1998), (Goferman et al., 2010), (Cheng et al., 2011), (Perazzi et al., 2012), or using spectral features like amplitude, phase spectrum (Hou and Zhang, 2007), (Achanta et al., 2009), (Li et al., 2013), (Schauerte and Rainer, 2012) and image energy in the spectral domain (Hou et al., 2012) or graph based method (Harel et al., 2006). Most of them have defined saliency as rarity of occurrence (or as a surprise) with respect to different local and global features. Color difference in CIELab space is the most distinctive feature which is used across most of the models. Our spectral clustering based rarity and spatial compactness measures of saliency exploit rarity of feature to extract salient regions in an image.

Spectral clustering ( $\mathrm{Ng}$ et al., 2001) is used in many different applications like, page ranking (Zhou et al., 2004), contour detection (Arbelaez et al., 2011), normalized cut (Shi and Malik, 2000) approaches. A recent paper (Yang et al., 2013) uses the ranking algorithm (Zhou et al., 2004) to find salient regions in images. We do not use any ranking technique (Zhou et al., 2004) or like any Normalized Cut approach, we do not cluster the descriptors obtained from the eigenvectors of the graph Laplacian. Instead, we find the rarity using these descriptors itself, since eigenvectors themselves carry information about the superpixels. 
Along with the above measures, we exploit the concept of background prior which is same as boundary prior and connectivity prior (Wei et al., 2012) concept. In this paper, background refers to the nonsalient spatial locations in the image. The main idea is that, the distance between background patches will be less and that between background and a salient patch will be more. Recent, cognitive science literature (Tatler, 2007) gives the evidence of boundary prior and shows human fixation happens mostly at the center. This motivates our second component of saliency detection where we statistically model the boundary patches of an image and use them as background prior in a complete unsupervised formulation to detect saliency. We call our proposed method which is based on Graph-based Rarity, Spatial Compactness and Background Prior, as PARAM (background Prior And RArity for saliency Modeling).

\section{RELATED WORK}

Bottom up saliency models are mostly inspired by neurophysiology, which adapt the concepts of feature integration theory (FIT) (Tre, ) and visual attention (Koch and Ullman, 1987). Itti et al.'s (Itti et al., 1998) model uses three features, color, intensity and orientation, like the simple cells in primary visual cortex. Most computational models are based on either spatial or spectral processing. Spatial models use different local or global features, like color, intensity, spatial distance, or a combination. Spectral models use a spectral domain analysis of the image and inherently use global features.

Among spatial models, Goferman et al. (Goferman et al., 2010) model saliency using both local lowlevel features and global considerations, as well as visual organization rules and high level features. They have taken overlapping patches at different scales and modeled saliency as distance in color, inversely weighted by distance in position among the patches. As a result, edges of the salient regions are highlighted more. Cheng et al. (Cheng et al., 2011) have proposed a region-wise contrast based method to compute saliency and uses GrabCut algorithm to give a refined saliency cut. Being a global contrast based method, it works well for only large-scale salient regions. Perazzi et al. (Perazzi et al., 2012) divide the image into superpixels, computes saliency using uniqueness and distribution properties and upsamples it to gives a smooth, pixel-wise accurate salient region. It gives a good precision for focused, large salient regions but fails for small salient regions and also for cluttered background. A recent method
(Yang et al., 2013) uses a spectral clustering based ranking algorithm to rank the superpixels according to their saliency, or precisely get the saliency probability values. Harel et al.'s model (Harel et al., 2006) although fails to detect the entire salient object, works comparatively well for multiple salient regions. But, it gives a blurred map with less precision.

Among spectral analysis models, Hou and Zhang (Hou and Zhang, 2007) represent a log spectrum and Gaussian smoothed inverse Fourier transformed spectral component for saliency. They use only the phase information and thus works better for small salient regions in an uncluttered background (Li et al., 2013). Achanta et al. in their model (Achanta et al., 2009) first omit the very high frequency components as those correspond to background texture or noise artifacts and then computes saliency as the distance from mean color in Lab color space. A more advance model (Li et al., 2013) uses hypercomplex Fourier transform (HFT) over different features like (Itti et al., 1998) and does a spectrum scale space analysis. Optimal scale is detected by minimizing an entropy, with saliency as probability maps. It gives good results for images with different sizes of salient regions with varying background, but results are blurred and fails to give accurate object boundary. Hou et al. in (Hou et al., 2012) prove that Inverse Discrete Cosine Transform (IDCT) of the sign of DCT of an image, concentrates the image energy at the location of spatially sparse foreground. This holds good for only small and sparse salient regions.

In this paper, we propose a novel unsupervised formulation of saliency measure using appropriate features for discriminating the salient region from the background. Features are based on color and spatial distance of superpixels. Graph based (spectral clustering) rarity approach uses eigenvectors of the Laplacian of the affinity graph. The spatial compactness term is a modified version of the distribution term of (Perazzi et al., 2012). The other component is color divergence with respect to the patches at the border of an image. (Wei et al., 2012) uses the concept in a semi-supervised algorithm which requires manual intervention. We statistically model the boundary patches using Gaussian Mixture Model (GMM), and find the distance of all the patches from the modeled background colors in Lab color space. Integration of these priors gives the saliency map. 


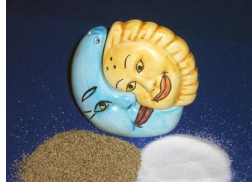

(a)

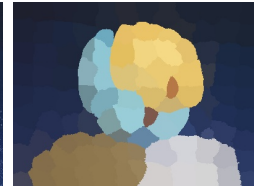

(b)

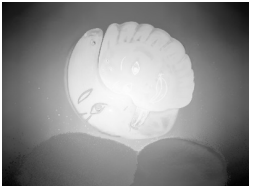

(c)

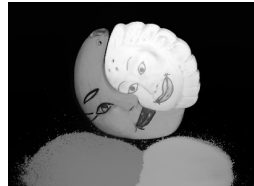

(d)

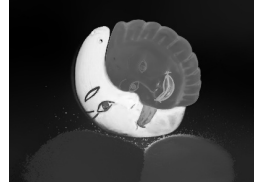

(e)

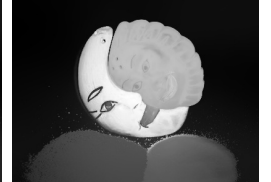

(e)

Figure 1: (a) Image; (b) Superpixels; (c)Saliency by Graph-based rarity; (d) Spatial compactness; (d) Background prior and (e) Proposed Saliency Map.

\section{BRIEF OVERVIEW OF OUR APPROACH}

\subsection{Abstract the Image into Superpixels}

We first break our image into superpixels using SLIC superpixel (Achanta et al., 2010) method which makes our method computationally fast. All computations are hence performed on superpixels which are much lesser in number than the set of pixels. Each superpixel is represented by a 5-D vector $\{1 \mathrm{abxy}\}$. Thus each patch has its specific color and position.

\subsection{Graph-based Spectral Rarity}

We use the eigenvectors of the normalized Laplacian matrix of the affinity graph with superpixels as nodes. If we look closely, the laplacian matrix provides a measure of the fraction of time a free random walker would spend at each node and what are the most preferable nodes to go from a particular node, considering the edge weights as cost of moving from one node to the other. Hence, as also mentioned by (Arbelaez et al., 2011), this carries information about the edges in the image. If a random walker has little probability to move from a particular node to another, there is an edge in the image between the two superpixels. The descriptor extracted from the eigenvectors of the normalized Laplacian matrix, when using superpixels as nodes, would capture the corresponding coarse texture information. Hence, local and global rarity based on these descriptor would give a measure of saliency which takes rarity of textures into account.

\subsection{Spatial Compactness}

We exploit the fact that a salient object would be spatially compact and the background colors will be distributed over the whole image (Goferman et al., 2010) (Hou and Zhang, 2007). As, human eye can fixate at only one position and vision is centre surround, spatial compactness is an important characteristics of an object to become salient. So, the color belonging to the salient object will be spatially clustered together. Whereas, colors belonging to background will have high spatial variance. Hence, we use spatial variance of color or color compactness as a measure of saliency detection. The less the spatial variance more compact the object is and thus more salient.

\subsection{Background Prior}

However, rarity of feature alone is not sufficient to detect saliency, as some previous methods in literature (Perazzi et al., 2012)(Cheng et al., 2011) show. We exploit the concept of boundary prior (Wei et al., 2012), which comes from the natural fact that boundary of an image would be mostly occupied by background (Tatler, 2007). Moreover, background will be mostly spatially distributed but homogeneous (in parts, say, the sky above and the grass below, for a natural scene) which results in compact clusters in color (feature) space. Hence, distance between these background patches will be less, but background and fore-ground salient patches will be high, in 3-D Lab color space. However, occasionally a part of the salient object may exists at the boundary. Hence, it is not justified to consider all the boundary patches as background. To solve this, we statistically model the boundary patches using GMM. Here, Gaussian modes with large number of pixels, having a large value of mixture coefficient, will generally model the background colors. Whereas, some Gaussian modes which model the few salient object patches, present at the image boundary, will naturally have low mixture coefficient. Hence, we exploit Mahalonobis distance in color space, between the image patches and these modes, weighted by the corresponding mixture coefficients, to computed a good measure of saliency.

\subsection{Pixel Accurate Saliency}

Finally, we combine the saliency measures yielding a granulated saliency map at superpixel level. To get a pixel accurate saliency map we use the up-sampling technique proposed by (Dolson et al., 2010)(Perazzi et al., 2012). 
Results of individual components are illustrated in the Figure 1 and shows it finally produces an improved saliency map.

\section{ALGORITHM FOR SALIENCY MAP ESTIMATION}

We formulate two new measures of saliency detection, using graph-based rarity, spatial compactness of color and statistical model of boundary colors. The overall process of saliency computation is described in the following subsections:

\subsection{Pre-processing}

We first represent the image using superpixels by exploiting the concept of SLIC superpixel segmentation (Achanta et al., 2010) in five-dimensional \{labxy\} space. We input the number of clusters as 400 , for all the experiments, to the SLIC superpixel algorithm which yields $N$ superpixels. The benefit of SLIC segmentation is that, it produces compact homogeneous color patches as clusters. This helps the next stages of our algorithm. Each superpixel, $i$ has color in CIELab space $c_{i}$ and position $p_{i}$. In the following subsection, we describe the measures for saliency computation.

\subsection{Saliency Computation}

Our measure of saliency has broadly two components for salient object detection. The first one is given by graph-based spectral rarity and spatial compactness of the salient object. This approach exploits rarity of feature. Whereas, the second component of saliency detection utilizes the concept of boundary prior and connectivity prior (Wei et al., 2012).

\subsubsection{Graph-based Spectral Rarity}

Given an image we define a graph $\mathrm{G}=(\mathrm{V}, \mathrm{E})$ whose nodes are the superpixels and edges $\mathrm{E}$ are weighted by an affinity matrix $\mathrm{W}=\left[w_{i j}\right]_{N X N}$. Let $\mathrm{D}=\operatorname{diag}\left\{d_{11}, \ldots, d_{N N}\right\}$, where $d_{i i}=\sum_{j} w_{i j}$. Then Laplacian of the graph $\mathrm{G}$, can be given by $\mathrm{L}=I-$ $D^{-\frac{1}{2}} W D^{-\frac{1}{2}}$. Let, $\left\{v_{1}, \ldots, v_{k}\right\}$ are the eigenvectors corresponding to largest $k$ eigenvalues of $\mathrm{L}$. We form the matrix $X_{N \times k}$ by stacking the eigenvectors in columns. Now we take normalized row vectors of $X_{N \times k}$ as the descriptor for each superpixel. Let the $k$-dimensional descriptors are $\left\{x_{1}, \ldots, x_{N}\right\}$ and position of superpixel $i$ is $p_{i}$. We find rarity of the $i^{t h}$ superpixel using the following formulation,

$$
r_{i}=\sum_{j=1}^{N}\left\|x_{j}-x_{i}\right\|^{2} \exp \left(-k_{r}\left\|p_{j}-p_{i}\right\|^{2}\right)
$$

where, ||.|| implies Euclidean distance. $k_{r}$ is the factor that controls how much local the rarity is. If $k_{r}$ is infinite, it becomes a global rarity measure. $k_{r}$ is set to 8.0 in all the experiments. We take the weight between two nodes, $w_{i j}=\exp \left(-\left\|c_{i}-c_{j}\right\|^{2}\right), i, j \in V$. Figure 1 (b) shows the saliency map generated using only $r_{i}$ as saliency probability of $i^{t h}$ superpixel.

\subsubsection{Spatial Compactness}

We define spatial variance of color $\left(v_{i}\right)$ of a superpixel $i$, with color in CIELab space $c_{i}$ and position $p_{i}$ as, how much similar colored patches are distributed over the image. A salient color is expected to be spatially compact and thus will be close to the spatial mean position of the particular color (Perazzi et al., 2012). Thus, $v_{i}$ is computed as,

$$
v_{i}=\sum_{j=1}^{N}\left\|p_{j}-\mu_{i}\right\|^{2} \cdot \exp \left(-k_{c}\left\|c_{j}-c_{i}\right\|^{2}\right)
$$

where, $\mu_{i}$, the weighted mean position of color $c_{i}$, gives the mean position of a particular color, $c_{i}$, weighted by the difference in color with other similar colored patches, as

$$
\mu_{i}=\frac{\sum_{j=1}^{N} p_{j} \cdot \exp \left(-k_{c}\left\|c_{j}-c_{i}\right\|^{2}\right)}{\sum_{j=1}^{N} \exp \left(-k_{c}\left\|c_{j}-c_{i}\right\|^{2}\right)}
$$

$k_{c}$ controls the sensitivity of color similarity while computing their spatial mean position. $k_{c}$ is set as in (Perazzi et al., 2012) in all the experiments. High value of $k_{c}$ implies that, only when the colors of the patches are very similar, it would contribute to the computation of $\mu$ for that particular color.

If the spatial variance of color for superpixel $i$ is less, it corresponds to a salient region, and not the background, as background colors are generally dispersed over the entire image. Thus, for a salient superpixel $i$, its mean $\left(\mu_{i}\right)$ will be spatially near to, $p_{i}$ and also to all the $p_{j}$ s belonging to similar colored patches in 2-D spatial space. And only the patches, for which $c_{j} \simeq c_{i}$ will contribute to the term $v_{i}$. So, for a salient patch $i, v_{i}$ will be small as $p_{j}$ s are close to $\mu_{i}$ making ||$p_{j}-\mu_{i} \|$ small $\forall j \mid c_{j} \simeq c_{i}$. Hence, The lower the value of $v_{i}$, the more salient is the region $i$.

Hence, our first component of saliency for $i^{\text {th }}$ superpixel using feature rarity is given as,

$$
F_{i}=\exp \left(-k \cdot v_{i}\right) \cdot r_{i}
$$




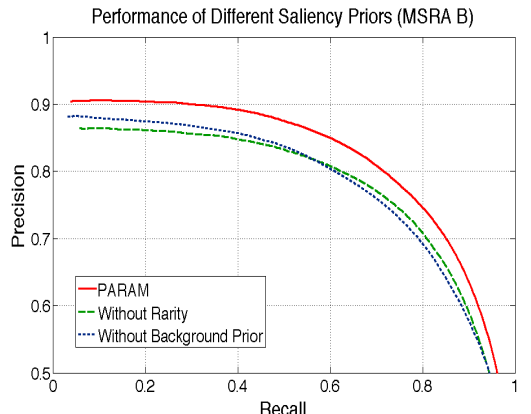

(a)

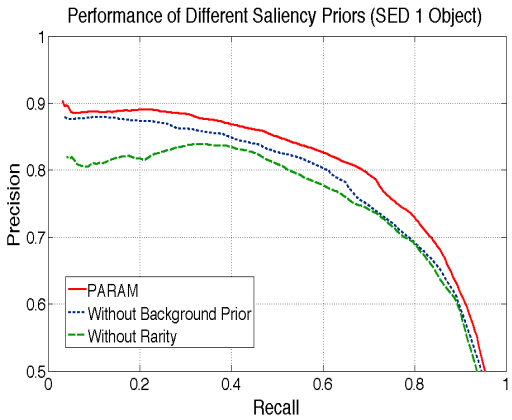

(b)

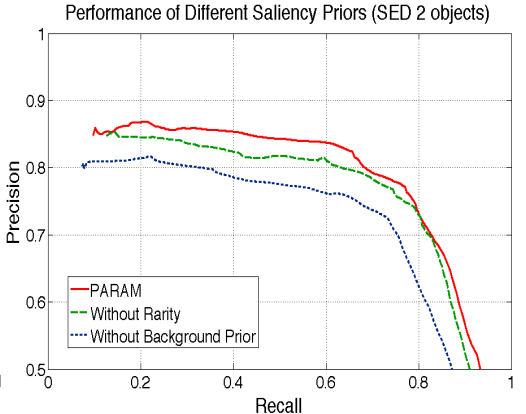

(c)

Figure 2: Performance curve illustrating the importance of the different components of our method (PARAM) for saliency computation using Precision vs Recall metric on: (a) MSRA B; (b) SED1 and (c) SED2 Datasets.

Figure 1 (c) shows the saliency map generated using only $\exp \left(-k \cdot v_{i}\right)$ as saliency probability of $i^{t h}$ superpixel. Large value of $F_{i}$ indicates greater saliency. $k$ is the scale of the exponent and set to 3 in all the experiments, as in (Perazzi et al., 2012).

\subsubsection{Background Prior}

The above feature rarity based method is not enough to find salient object in all different types of images, specially with small or more than one salient objects. We assume that boundary superpixels are less likely to be salient and recent studies in cognitive science (Tatler, 2007) reveals the same. This criteria is derived from prior observation of samples from various benchmark datasets, and requirements of saliency in many application domains (CBIR, object recognition, target acquisition etc.). We model the boundary superpixels using a Gaussian Mixture Model (GMM) in CIELab-color space and find the Mahalanobis distance $\left(D_{M}\right)$ of all the superpixels from the Gaussians means. The more a superpixel differs in Lab-color space from the boundary superpixels, more is its saliency. Whereas, background superpixels are mostly homogeneous and thus distance of background patches from these GMM means will lesser. Again, boundary is mostly occupied by nonsalient background superpixels. So, more the value of mixture coefficient $\left(\pi_{j}\right)$ of a GMM component, more likely that the component refers to a non-salient color.

Following above, the second component of saliency measure of $i^{\text {th }}$ superpixel, using background prior is formulated as,

$$
B_{i}=\sum_{j=1}^{K} \pi_{j} . D_{M}\left(c_{i}, \mu_{G j}\right)
$$

where, $D_{M}(x, y)$ denotes Mahalanobis distance between $x$ and $y, c_{i}$ is the color of $i^{t h}$ superpixel, $\mu_{G j}$ is the mean of $j$ th Gaussian mode, $\pi_{j}$ is the weight or mixture coefficient of the $j^{\text {th }}$ Gaussian and $\mathrm{K}$ is the number of GMM components used to model the distribution of the boundary superpixels in CIELab space. We dynamically compute the optimal value of $K$ maximizing the cluster compactness of the boundary patches. Figure 1 (d) shows the saliency map generated using $B_{i}$ as saliency probability of $i^{\text {th }}$ superpixel.

\subsection{Saliency by Up-sampling to Image Resolution}

Saliency of each pixel is taken as a weighted linear combination of saliency of its surrounding image elements, $S_{j}$, using the idea proposed by (Dolson et al., 2010). In our work, $S_{j}$, the saliency value of $j^{\text {th }}$ patch, is sum of $F_{j}$ and $B_{j}$ and we use the same formulation as used in (Perazzi et al., 2012).

Figure 2 shows the contribution of the saliency priors. It shows the performances by excluding $r_{i}$ from eq. 4 and without background prior concept (eq. 5), that is only exploiting eq. 4, along with final saliency map (PARAM), on different datasets (for details of experimentation see Section 5.2).

\section{RESULTS AND PERFORMANCE EVALUATION}

\subsection{Datasets}

We evaluate the performance of our proposed method (PARAM) using the following two benchmark datasets. 


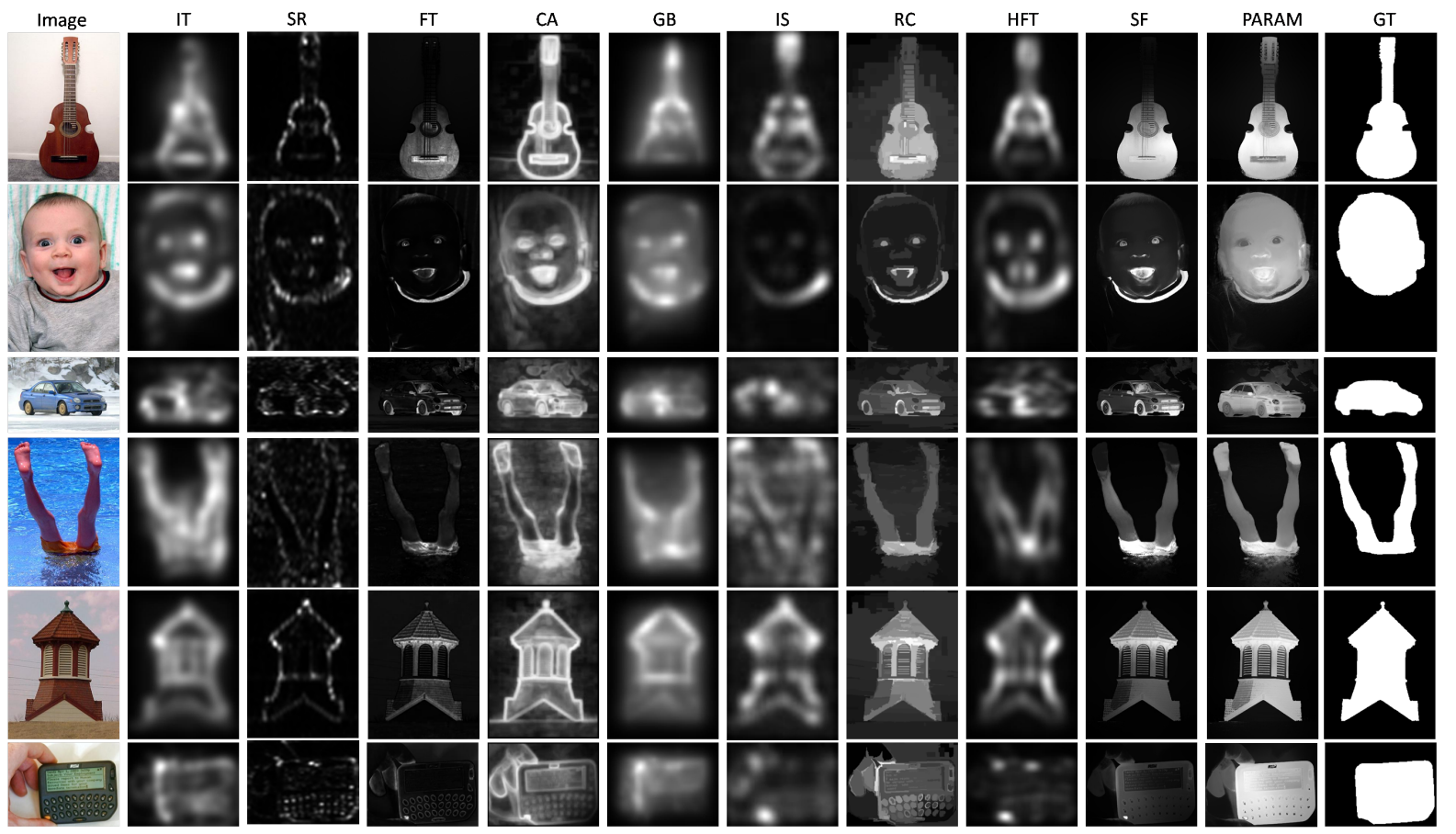

Figure 3: Visual comparison of the results of nine state-of-the-art methods along with our proposed method (PARAM) of saliency estimation on six different samples of MSRA B dataset. PARAM consistently performs better for different types of images including indoor, outdoor natural scenes, when compared with the ground truth given in the last column.

\subsubsection{MSRA B $\mathbf{B}^{1}$}

MSRA B has 5000 images with their ground truth masks as given in the papers, (Achanta et al., 2009) and (Jiang et al., 2013). Images are of numerous kind including indoor, outdoor natural scenes, humans, animals with different types of contrast and color variance. This makes the dataset diverse and challenging.

\subsubsection{SED $^{2}$}

Segmentation Evaluation Dataset (SED) has two parts, SED1 and SED2. SED1 has 100 images with a single salient object. SED2 images has 100 images with 2 different salient objects of different size and color. Ground truth masks for all the images are publicly available.

\subsection{Experimentation}

We compare the performance of our proposed method, PARAM, with 9 state-of-the-art methods, IT (Itti et al., 1998), SR (Hou and Zhang, 2007), CA (Goferman et al., 2010), FT (Achanta et al., 2009),

\footnotetext{
${ }^{1}$ http://research.microsoft.com/en-us/um/people /jiansun/SalientObject/salient_object.htm

${ }^{2} \mathrm{http}: / /$ www.wisdom.weizmann.ac.il/ $\sim$ vision/ Seg_Evaluation_DB/dl.html
}

RC (Cheng et al., 2011), IS (Hou et al., 2012), GB (Harel et al., 2006), HFT (Li et al., 2013), SF (Perazzi et al., 2012).

Figures 3 - 6 show results of these 9 different stateof-the-art saliency detection methods along with our proposed method, PARAM. Results in Figure 3 visually illustrates that the saliency map provided by our method (PARAM) is closest to the ground truth (denoted by GT) and highlights the overall salient object uniformly, giving better result than the existing stateof-the-art methods.

\subsubsection{Quantitative Performance Evaluation}

We quantitatively evaluate the performance of our method (PARAM) using precision, recall rate similar to the (Achanta et al., 2009), (Cheng et al., 2011), (Hou and Zhang, 2007). We generate the precisionrecall curve by producing binary maps at different thresholds similar to (Achanta et al., 2009). We have compared our method with all the above mentioned 9 state-of-the-art methods. We do not compare with (Jiang et al., 2013), as being a training based method it has output for only 2000 images. Our method, PARAM clearly out-performs all the methods on MSRA B (Figure 4 (a)) and SED1 (Figure 4 (b)) datasets. On SED2 (Figure 4 (c)) dataset, PARAM is not a clear winner. This is mainly due to the occa- 


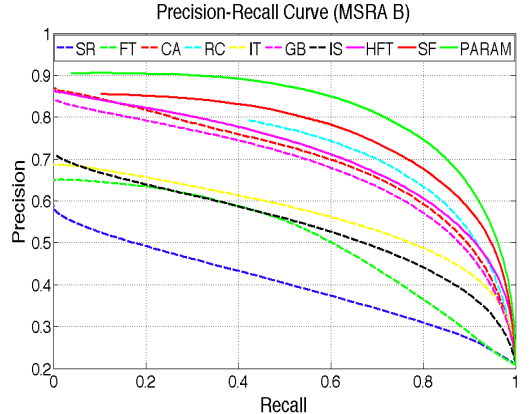

(a)

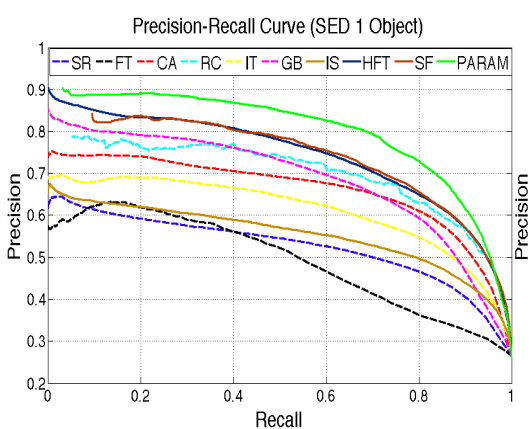

(b)

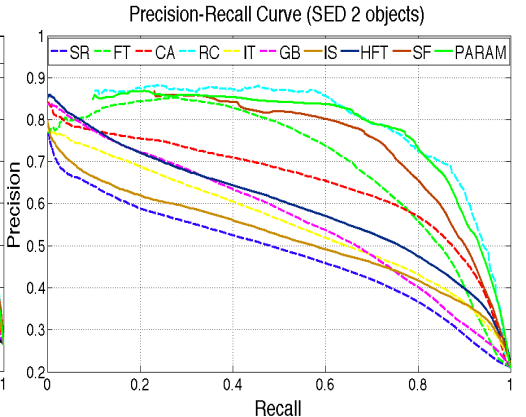

(c)

Figure 4: Performance analysis of 9 different state-of-the-art methods along with our proposed method (PARAM) using Precision vs Recall metric on: (a) MSRA B; (b) SED1 and (c) SED2 Datasets. It shows that our method performs over all the best on MSRA B as well as both the SED datasets. This figure is best viewed in color.

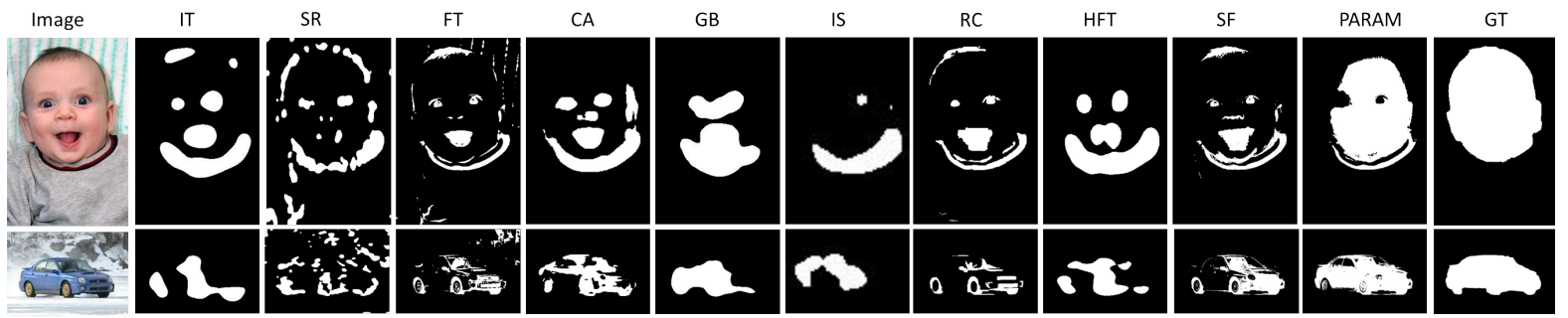

Figure 5: Visual comparison of the Adaptive Cut binary maps of the nine state-of-the-art methods and our proposed method (PARAM) on two samples of MSRA B dataset, with the ground truth as given in the last column.

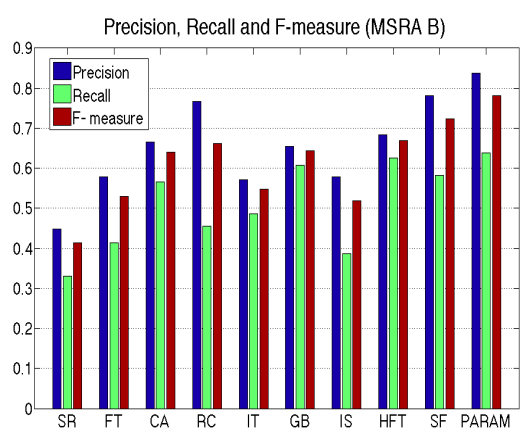

(a)

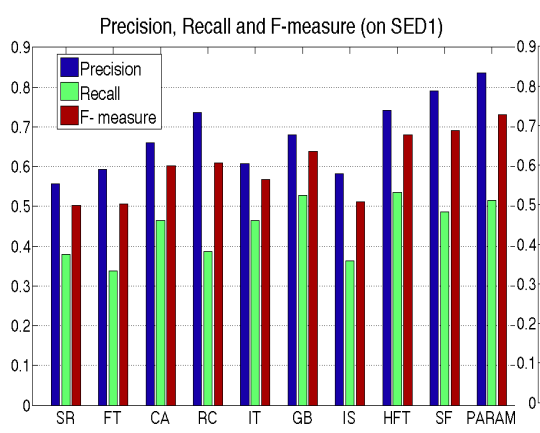

(b)
Precison, Recall and F-measure (on SED2)

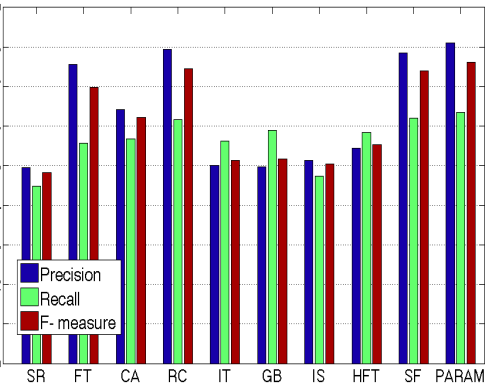

(c)

Figure 6: Precision, Recall \& F-measure using adaptive cut on (a) MSRA B; (b) SED1 and (c) SED2 datasets, show that our method (PARAM) performs better than all the 9 state-of-the-art methods for all the datasets.

Table 1: Average runtime (in seconds per image) of different competing methods.

\begin{tabular}{|c|c|c|c|c|c|c|c|c|c|}
\hline Method & IT & FT & GB & CA & RC & IS & HFT & SF & PARAM \\
\hline Time (s) & 0.41 & 0.13 & 1.63 & 128.05 & 0.21 & 2.20 & 0.76 & 0.23 & 0.23 \\
\hline
\end{tabular}

sional presence of two objects only on the boundary. Such a scenario is not biologically plausible to become salient for human vision.

We take the adaptive threshold as twice of average saliency and create a binary map, which is proposed as Adaptive Cut in (Achanta et al., 2009). Figure 5 shows the binary maps or adaptive cuts which are generated using adaptive threshold, from the saliency maps obtained from the 9 different state-of-the-art and our proposed method, PARAM. From these binary maps we calculate specific values of precision, recall and the f-measure as in (Achanta et al., 2009), for the 9 methods along with PARAM. The bar charts in Figure 6 show that PARAM produces the best result for all the three performance measures: Precision, Recall and F-measure. 


\subsubsection{Efficiency}

Although our method has different saliency priors, it is time efficient and can be easily used as preprocessing step for different applications. This is mainly due to the fact that the saliency computation by our method is performed on image patches or superpixels which are much lesser in number than the set of pixels. Moreover, parallel computation of the priors is also possible. We compare the running time of our implementation (in $\mathrm{C}++$ ) with other competing methods. We use Matlab implementation from authors for (Itti et al., 1998), (Goferman et al., 2010), (Achanta et al., 2009), (Li et al., 2013), (Harel et al., 2006), (Hou et al., 2012) and C++ implementation of (Cheng et al., 2011), (Perazzi et al., 2012) on a intel core 2 extreme $3.00 \mathrm{GHz} \mathrm{CPU}$ with $4 \mathrm{~GB}$ RAM. Table 1 lists the average running time of 8 competing methods along with PARAM. For (Hou and Zhang, 2007) we get the results from the publicly available executable of (Cheng et al., 2011), and we do not have the time efficiency information for the same. The work proposed in (Achanta et al., 2009) is the fastest, but performs much inferior (refer Figures 3 - 6).

\section{CONCLUSIONS}

We have presented a bottom-up saliency estimation method for images using low level cues. We have proposed a novel graph-based feature rarity computation, utilizing the concepts of spectral clustering ( $\mathrm{Ng}$ et al., 2001). It shows that eigenvectors of Laplacian of the affinity matrix of the graph, taking image elements as node gives good measure of rarity. Again, we exploit spatial compactness of color and we use the cue of boundary prior by statistically modeling the background in color space. We show, both qualitatively (Figure 1) and quantitatively using PrecisionRecall metric (Figure 2), that these components compliment each other. We also give a comparative study of the performance of our method with 9 state-of-theart methods, using three different measures of evaluation on two popular real-world benchmark datasets. Since, our method is not just restricted to global spatial feature rarity, but also utilizes the boundary cue as well as spectral clustering based feature rarity, it gives better performance and in most of the cases accurately detects the salient object.

\section{REFERENCES}

A feature-integration theory of attention. Cognitive Psychology, 12(1).

Achanta, R., Hemami, S., Estrada, F., and Susstrunk, S. (2009). Frequency-tuned salient region detection. In CVPR.

Achanta, R., Shaji, A., Smith, K., Lucchi, A., Fua, P., and Susstrunk, S. (2010). SLIC Superpixels. Technical report, EPFL.

Arbelaez, P., Maire, M., Fowlkes, C., and Malik, J. (2011). Contour detection and hierarchical image segmentation. TPAMI, 33(5):898-916.

Cheng, M.-M., Zhang, G.-X., Mitra, N. J., Huang, X., and $\mathrm{Hu}$, S.-M. (2011). Global contrast based salient region detection. In $C V P R$.

Dolson, J., Jongmin, B., Plagemann, C., and Thrun, S. (2010). Upsampling range data in dynamic environments. In $C V P R$.

Goferman, S., Zelnik-manor, L., and A.Tal (2010). Contextaware saliency detection. In CVPR.

Harel, J., Koch, C., and Perona, P. (2006). Graph-based visual saliency. In NIPS, pages 545-552.

Hou, X., Harel, J., and Koch, C. (2012). Image signature: Highlighting sparse salient regions. TPAMI, 34(1).

Hou, X. and Zhang, L. (2007). Saliency detection: A spectral residual approach. In $C V P R$, pages 1-8.

Itti, L., Koch, C., and Niebur, E. (1998). A model of saliency-based visual attention for rapid scene analysis. TPAMI, 20(11).

Jiang, H., Wang, J., Yuan, Z., Wu, Y., Zheng, N., and Li, S. (2013). Salient object detection: A discriminative regional feature integration approach. In CVPR.

Koch, C. and Ullman, S. (1987). Shifts in selective visual attention: Towards the underlying neural circuitry. In Matters of Intelligence, volume 188. Springer Netherlands.

Li, J., Levine, M. D., An, X., Xu, X., and He, H. (2013). Visual saliency based on scale-space analysis in the frequency domain. TPAMI, 35(4).

Ng, A. Y., Jordan, M. I., and Weiss, Y. (2001). On spectral clustering: Analysis and an algorithm. In NIPS, pages 849-856.

Perazzi, F., Krahenbuhl, P., Pritch, Y., and Hornung, A. (2012). Saliency filters: Contrast based filtering for salient region detection. In $C V P R$.

Schauerte, B. and Rainer, S. (2012). Quaternion-based spectral saliency detection for eye fixation prediction. In $E C C V$, pages 116-129.

Shi, J. and Malik, J. (2000). Normalized cuts and image segmentation. TPAMI, 22(8):888-905.

Tatler, B. W. (2007). The central fixation bias in scene viewing: Selecting an optimal viewing position independently of motor biases and image feature distributions. Journal of Vision, 7(14).

Wei, Y., Wen, F., Zhu, W., and Sun, J. (2012). Geodesic saliency using background priors. In $E C C V$.

Yang, C., Zhang, L., Lu, H., Ruan, X., and Yang, M.-H. (2013). Saliency detection via graph-based manifold ranking. In $C V P R$.

Zhou, D., Weston, J., Gretton, A., Bousquet, O., and Schlkopf, B. (2004). Ranking on data manifolds. In NIPS. 